J. Dairy Sci. 99:1595-1604

http://dx.doi.org/10.3168/jds.2015-10353

(C) American Dairy Science Association ${ }^{\circledR}, 2016$.

\title{
Sterol regulatory element binding protein-1 (SREBP-1)c promoter: Characterization and transcriptional regulation by mature SREBP-1 and liver $X$ receptor $\alpha$ in goat mammary epithelial cells
}

\author{
H. F. Xu, ${ }^{,}$J. Luo, ${ }^{* 1}$ H. P. Wang, † H. Wang, ${ }^{*}$ T. Y. Zhang, ${ }^{*}$ H. B. Tian, ${ }^{*}$ D. W. Yao, ${ }^{*}$ and J. J. Loor ${ }^{1}$ \\ *Shaanxi Key Laboratory of Molecular Biology for Agriculture, College of Animal Science and Technology, Northwest A \& F University, Yangling, \\ 712100, PR China \\ †College of Animal Sciences, Zhejiang University, Hangzhou, 310058, PR China \\ ¥Mammalian NutriPhysioGenomics, Department of Animal Sciences and Division of Nutritional Sciences, University of Illinois, Urbana 61801
}

\section{ABSTRACT}

Sterol regulatory element binding protein-1 (SREBP-1) is a key transcription factor that regulates lipogenesis in rodent liver. Two isoforms (SREBP-1a and SREBP-1c) of SREBP-1 are transcribed by an alternative promoter on the same gene (SREBF1), and the isoforms differ only in their first exon. Although the regulatory effects of SREBP-1 on lipid and milk fat synthesis have received much attention in ruminants, $S R E B P-1 c$ promoter and its regulatory mechanisms have not been characterized in the goat. In the present study, we cloned and sequenced a 2,012-bp fragment of the SREBP-1c 5'-flanking region from goat genomic DNA. A luciferase reporter assay revealed that SREBP-1c is transcriptionally activated by the liver X receptor $\alpha(\mathrm{LXR} \alpha)$ agonist T0901317, and is decreased by SREBP-1 small interfering (si)RNA. A 5 ' deletion analysis revealed a core promoter region located -395 to $+1 \mathrm{bp}$ upstream of the transcriptional start site (TSS). Site-directed mutagenesis of LXR $\alpha$ binding elements (LXRE1 and LXRE2) and sterol regulatory elements (SRE1 and SRE2) revealed that the full effects of T0901317 require the presence of both LXRE and SRE. We also characterized a new SRE (SRE1) and demonstrated a direct role of SREBP-1 (autoloop regulation) in maintaining its basal transcription activity. Results suggest that goat $S R E B P-1 c$ gene is transcriptionally regulated by mature SREBP-1 (autoloop circuit regulation) and LXR $\alpha$ in goat mammary epithelial cells.

Key words: sterol regulatory element binding protein1c (SREBP-1c), liver $\mathrm{X}$ receptor $\alpha(\mathrm{LXR} \alpha)$, milk fat, transcription regulation

Received September 5, 2015.

Accepted October 30, 2015.

${ }^{1}$ Corresponding authors: luojun@nwsuaf.edu.cn and jloor@illinois. edu

\section{INTRODUCTION}

The sterol regulatory element binding proteins (SREBP) are basic helix-loop-helix leucine zipper transcription factors synthesized as inactive precursors anchored in the membranes of the endoplasmic reticulum (Brown and Goldstein, 1997). Upon activation, the precursor protein undergoes a sequential 2 steps of proteolytic processing in Golgi apparatus, resulting in the release of $\mathrm{N}$-terminal active fragment (mature SREBP protein). Mature SREBP then translocates to the nucleus and binds to the sterol response element (SRE) in the promoter of target genes and to itself to regulate lipid metabolism (Eberlé et al., 2004; Espenshade, 2006). The identification of SRE in the promoter of SREBP-1c implies that mature SREBP-1 protein can bind to its own promoter and positively regulate its own expression through a characteristic "auto-loop circuit" regulation (Amemiya-Kudo et al., 2000; Lengi and Corl, 2010; Takeuchi et al., 2010).

Three SREBP members have been characterized; SREBP-1a and SREBP-1c are transcribed from a single gene, SREBF1 (human chromosome 17, mouse chromosome 11) but using different promoters, and they differ only in their first exons, whereas SREBP-2 is encoded by SREBF2 and mainly regulates cholesterol synthesis (Horton et al., 2002). The use of different promoters leads to a longer $\mathrm{NH}_{2}$-terminal transactivation domain in SREBP-1a than in SREBP-1c (Shimano et al., 1997), and it is highly expressed in cell lines and tissues with high capacity for cell proliferation, such as spleen and intestine. The SREBP-1c isoform is the predominant form expressed in liver, adipose tissue, and skeletal muscle in mice and humans (Shimomura et al., 1997).

Studies in rodents and dairy cows have provided strong evidence for SREBP-1 as a central player in the regulation of mammary fatty acid synthesis (Bionaz and Loor, 2008; Harvatine et al., 2009). Another important upstream regulator modulating SREBP-1c ex- 
pression transcriptionally is liver X receptor $\alpha(\mathbf{L X R} \mathbf{\alpha}$; Yoshikawa et al., 2001). The LXR $\alpha$ form heterodimers with retinoid X receptors (RXR) and then bind to specific DNA sequences (known as LXRE) in the promoter of target genes. Studies to date have identified LXRE in the promoter of cow, mouse, and human SREBP-1c gene, and these LXRE elements are demonstrated to be required for the full activation of SREBP-1 expression upon treatment with LXR $\alpha$ agonist (T0901317), indicating a direct regulation of SREBP-1c by LXR $\alpha$ (Repa et al., 2000; Yoshikawa et al., 2001; Lengi and Corl, 2010).

Goat milk represents an important source of nutrients for humans, but few studies have examined the role of goat SREBP-1c transcription regulation on milk fatty acid synthesis and there is no molecular information on the SREBP-1c promoter. Thus, the present study was designed to characterize the goat SREBP-1c promoter and to investigate its transcriptional regulation mechanism via LXR $\alpha$ and SREBP-1 in goat mammary epithelial cells (GMEC).

\section{MATERIALS AND METHODS}

The Animal Care and Use Committee of the Northwest A\&F University (China) approved all procedures involved in the use of live goats.

\section{Cloning of SREBP-1C Promoter Region and Bioinformatics Analysis}

The goat $S R E B P-1 c 5^{\prime}$-flanking sequence was amplified from goat blood genomic DNA using PrimeSTAR HS DNA Polymerase (Takara Bio Inc., Otsu, Japan) according to a previous publication (Lengi and Corl, 2010). Primers used for PCR amplification are listed in Table $1(-2,012$ to $+149 \mathrm{bp} ; 2,161 \mathrm{bp})$. The amplified product was cloned into the pMD19-T vector (Takara Bio Inc.) and sequenced (Sangon Biotech., Shanghai, China). Transcription factor binding sites of the SREBP-1c promoter were predicted using PATCH public 1.0 (http://www.gene-regulation.com/cgi-bin/ pub/programs/patch/bin/patch.cgi) and MatInspector (http://www.genomatix.de/).

\section{Deletion Analysis and Plasmid Construction}

The PCR primers were designed to hybridize at $-2,012,-1,549,-1,105,-395$, and -86 bp to generate the corresponding 5 '-flanking deletion plasmid derivatives using the common downstream primer at $+149 \mathrm{bp}$ ( 5 ' deletion primers are listed in Table 1$)$. The resulting amplicon was cloned into the pGL3-Basic vector (Pro- mega, Madison, WI) by using KpnI/HindIII (Takara Bio Inc.) enzyme sites. All the plasmids were confirmed by DNA sequencing.

\section{Site-Directed Mutagenesis}

Overlap extension PCR technology was used to generate site-directed mutants of the SREBP-1c promoter. Primers were designed to destroy the transcription factor binding sites by altering 3 nucleotides within each site (primer sequences are listed in Table 1). For the site mutant promoter, PCR reactions were conducted to generate 2 DNA fragments containing the designated mutations in the overlapping regions. Subsequently, 2 DNA fragments were pooled together as a PCR template to generate a full-length DNA fragment. The resulting amplicons were ligated to pGL3-basic vector after digestion with KpnI/HindIII (Takara Bio Inc.) to create mutant constructs. Each mutant construct was confirmed by DNA sequencing.

\section{Cell Culture and Transfection}

The GMEC were isolated and cultured as previously described (Wang et al., 2010; Lin et al., 2013; Shi et al., 2013). Before experiments, cells were seeded in a 48-well plate (Corning Inc., Corning, NY). Once grown to 80 to $90 \%$ confluence, GMEC were transiently transfected with $200 \mathrm{ng}$ of total DNA per well following the manufacturer's instructions for the X-treme GENE HP DNA Transfection Reagent [Roche (China) Ltd., Shanghai, China]. Renilla luciferase vector (pRL-TK) was used as an internal control to normalize transfection efficiency at a 25:1 ratio of pGL-SREBP1c and pRL-TK vector. For treatment with T0901317 after DNA transfection, medium supplemented with $1 \mu M$ T0901317 (dissolved in DMSO) was used, and the control group was treated with DMSO only. Each treatment was repeated in triplicate.

\section{Small Interfering RNA Transfection}

A gene-specific small interfering (si)RNA targeted for SREBP-1 was designed and synthesized commercially (Invitrogen, Carlsbad, CA). The siRNA sequence for SREBP-1 and its interfering efficiency have been published previously (Li et al., 2015). A scrambled siRNA was designed and provided by the same company and used as a negative control. After a 12-h transfection with DNA plasmid, siRNA were delivered to cells using Lipofectamine RNAiMAX (Invitrogen) at a final concentration of $100 \mathrm{n} M$ according to the manufacturer's instructions. 
Table 1. Primers used for cloning, deletion, and site-directed mutagenesis of sterol regulatory element binding protein-1c (SREBP-1c)

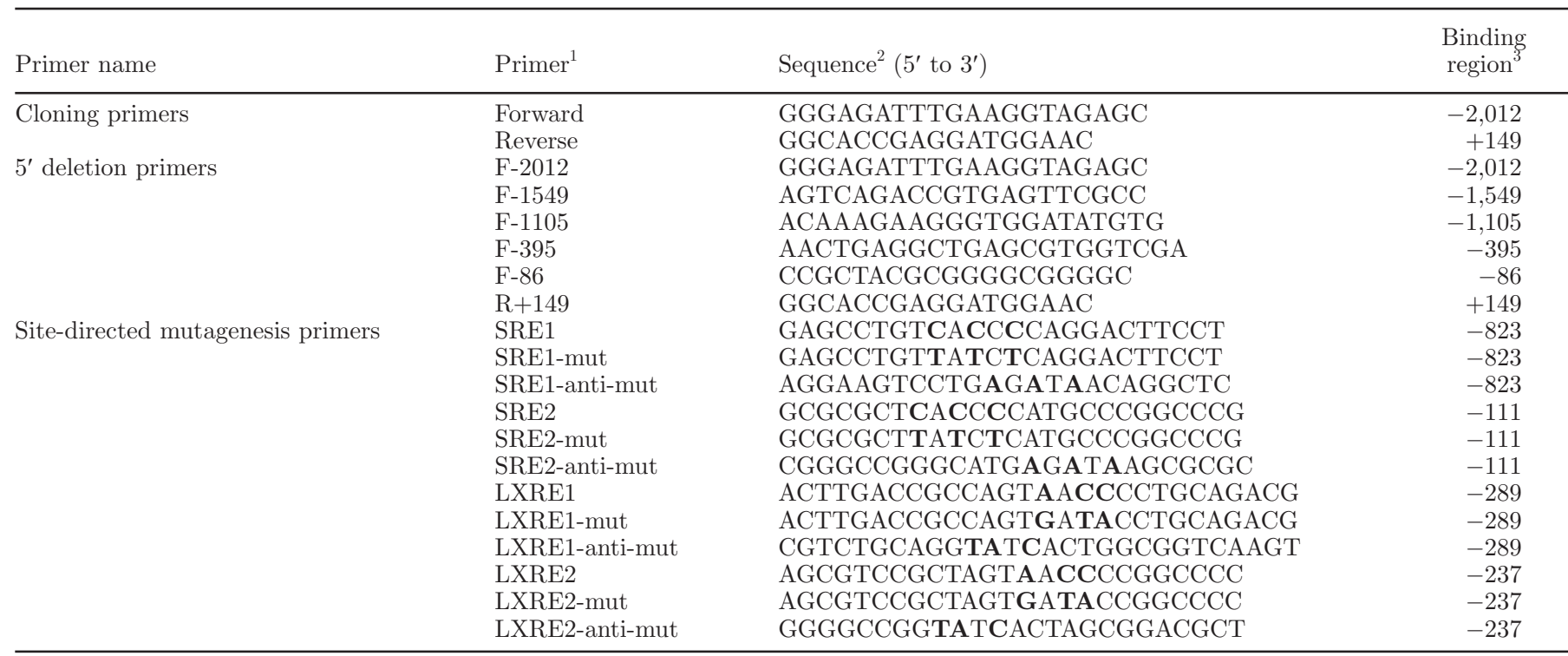

${ }^{1} \mathrm{SRE}=$ sterol response element; LXRE $=$ LXR (liver X receptor) response elements.

${ }^{2}$ Mutation letters are shown in bold font.

${ }^{3}$ Plus $(+)$ and minus $(-)$ indicate the number of nucleotides upstream $(-)$ and downstream $(+)$ from the transcriptional start site.

\section{Luciferase Assays}

After a 48-h incubation, cells were harvest and lysed for luciferase activity measurement. The relative luciferase activity was a ratio of firefly to Renilla luciferase activities assayed in a microplate reader by using the Dual-Luciferase Reporter Assay System (Promega) according to the manufacturer's instructions.

\section{Data Analysis}

All the treatments were conducted in triplicate and the results are presented as means \pm standard error of the mean. Statistical analyses were performed with the Student's $t$-test (paired and 2-tailed) and one-way ANOVA using SPSS 17.0 (SPSS Inc./IBM Corp., Chicago, IL). Differences were considered statistically significant when $P<0.05$.

\section{RESULTS}

\section{Cloning, Sequencing, and Characterization of the Goat SREBP-1c Promoter}

A 2,161-bp fragment of $5^{\prime}$-flanking sequence containing 2,012 bp upstream of the transcription start site $(+1)$ was amplified by PCR. An initiation codon ATG was located in exon 1, and a putative TATA box-like sequence was located at $-37 \mathrm{bp}$. Bioinformatics analysis showed transcription factor binding sites for SREBP-1
(SRE, -97 and $-808 \mathrm{bp}$ ), LXR (LXRE, -202 and $-271 \mathrm{bp}$ ), nuclear factor Y (NF-Y, -120 and -766 $\mathrm{bp})$, and transcription factor Sp1 $(-69,-87$, and -835 bp) (Figure 1).

\section{Detection of SREBP-1c Relative Luciferase Activity}

The GMEC transfected with pGL3-SREBP1c had an approximately 110-fold higher level of relative luciferase activity compared with pGL3-basic-transfected groups (Figure 2A), demonstrating strong activation of SREBP1c. By treating the pGL3-SREBP1c transfected group with SREBP-1-specific siRNA and T0901317 (agonist of LXR $\alpha$ ), we observed that siSREBP1 significantly reduced the relative luciferase activity of SREBP-1c (Figure 2B), whereas T0901317 treatment resulted in a dramatic increase of SREBP-1c promoter activity (Figure 2C).

\section{Luciferase Assay for Confirmation of the SREBP-1C Core Promoter Region}

To determine the core promoter region and characterize its essential cis-regulatory elements, we generated a series of $5^{\prime}$-flanking deletion constructs $(-2,012$ $\mathrm{bp} /+149 \mathrm{bp},-1,549 \mathrm{bp} /+149 \mathrm{bp},-1,105 \mathrm{bp} /+149 \mathrm{bp}$, $-395 \mathrm{bp} /+149 \mathrm{bp}$, and $-86 \mathrm{bp} /+149 \mathrm{bp}$ ) of SREBP-1c promoter and transfected them into GMEC. Results revealed no reduction in SREBP-1c promoter activity due to deletion of $-2,012 \mathrm{bp} /+149 \mathrm{bp},-1,549 \mathrm{bp} /+149 \mathrm{bp}$, 

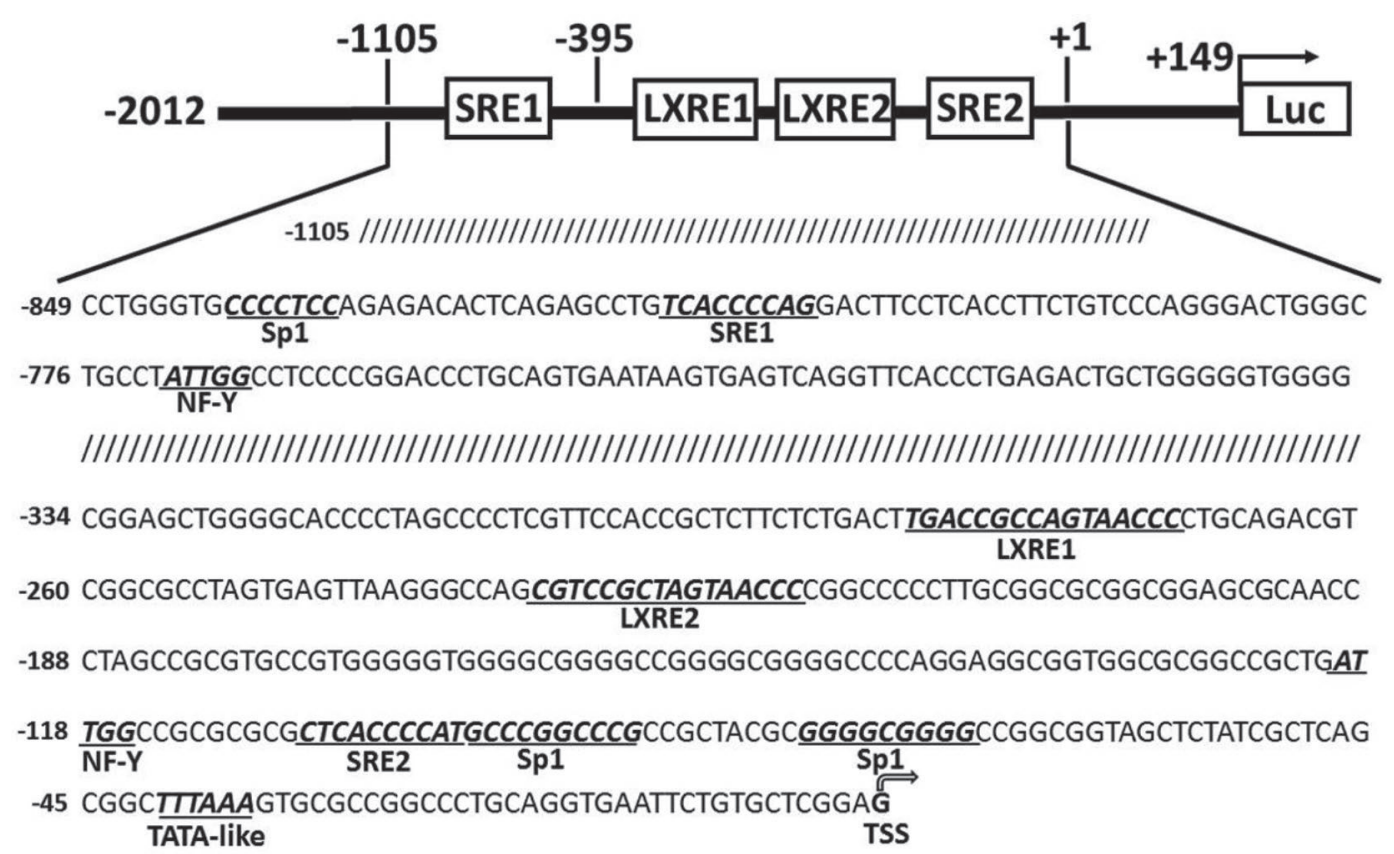

Figure 1. Schematic representation and characterization of goat sterol regulatory element binding protein-1c (SREBP-1c) promoter. The +1 indicates the transcriptional start site (TSS). Nucleotides of putative transcription factor binding sites and TATA box-like sequence are in boldface, italic, and underlined. SRE = sterol response element; LXRE = LXR (liver X receptor) response elements; NF-Y $=$ nuclear factor Y; $\mathrm{Sp} 1=$ transcription factor Sp1.

$-1,105 \mathrm{bp} /+149 \mathrm{bp}$, and $-395 \mathrm{bp} /+149 \mathrm{bp}$, whereas a dramatic decrease in SREBP-1c promoter activity was observed when deleting up to $-86 \mathrm{bp} /+149 \mathrm{bp}$ (Figure $3 \mathrm{~A})$. Except for the $-86 \mathrm{bp} /+149 \mathrm{bp}$ fragment, the LXR $\alpha$ agonist T0901317 induced the SREBP-1c promoter reporter gene significantly (Figure 3B). Taken together, these results suggest that the sequences between $-395 \mathrm{bp}$ and $+149 \mathrm{bp}$ contain the core promoter region of SREBP-1c and it bears the cis-functional elements required for its transcriptional activation in response to T0901317.

\section{SRE Are Responsible for Maintaining SREBP-1C Basal Transcription Activity}

Bioinformatics analysis revealed 2 SRE and 2 LXRE in the cloned fragment. One SRE (SRE2) and 2 LXRE (LXRE1 and LXRE2) are located in the core promoter region of SREBP-1c (Figure 1). To identify functional elements for the expression of SREBP-1c, we constructed a series of site-mutated expression vectors and transiently transfected them into GEMC for luciferase reporter assays. Results indicated that a single mutation of LXRE (mL1 or mL2) and a simultaneous mutation of both LXRE (mL1 mL2) did not reduce SREBP-1c promoter activity significantly. Mutations of SRE2 (mS2) decreased its promoter activity dramati- cally. And mutations of all sites ( $\mathrm{mL} 1 \mathrm{~mL} 2 \mathrm{mS} 2)$ nearly abolished promoter activity (Figure 4A).

To determine the role of SRE1 (located at $-808 \mathrm{bp}$ ) on SREBP-1c expression, we also generated a series of mutant reporter vectors containing $-1,105$ to +149 bp. No significant reductions of SREBP-1c promoter activity were detected after LXRE mutation (Figure 4B). However, single mutations of SRE ( $\mathrm{mS} 1$ or $\mathrm{mS} 2)$ and both mutations of SRE $(\mathrm{mS} 1 \mathrm{mS} 2)$ all reduced promoter activity significantly. Mutations of the 2 LXRE and 2 SRE (mL1 mL2 $\mathrm{mS} 1 \mathrm{mS} 2)$ resulted in very low activity (Figure 4B). Taken together, these results indicate that SRE are critical for maintaining SREBP-1c basal transcription activity, and LXRE play only a permissive role.

\section{LXRE Are Responsible for Full Activation of SREBP-1C in Response to T0901317}

To investigate the role of LXRE and SRE on the effects of T0901317 treatment on SREBP-1c promoter activity, we transiently transfected these site-directed mutant expression vectors into GMEC followed by treatment with DMSO and $1 \mu M$ T0901317 before luciferase reporter assays. The SRE mutations decreased but did not abolish the activation effects of T0901317 (Figure 5A and 5B). Similarly, mutations of 
A)

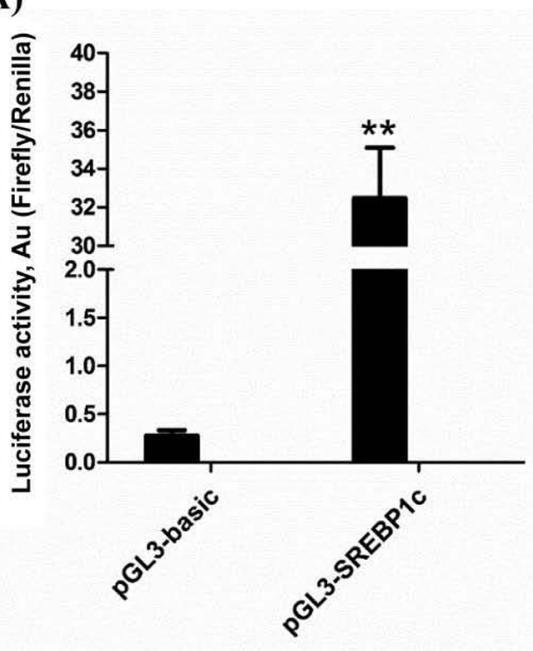

B)

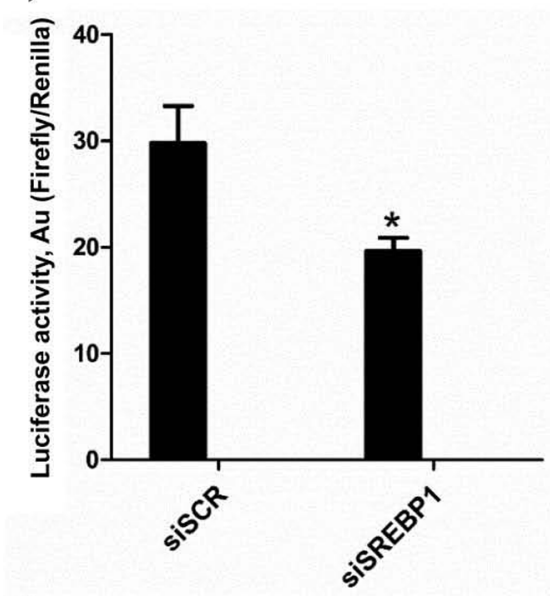

C)

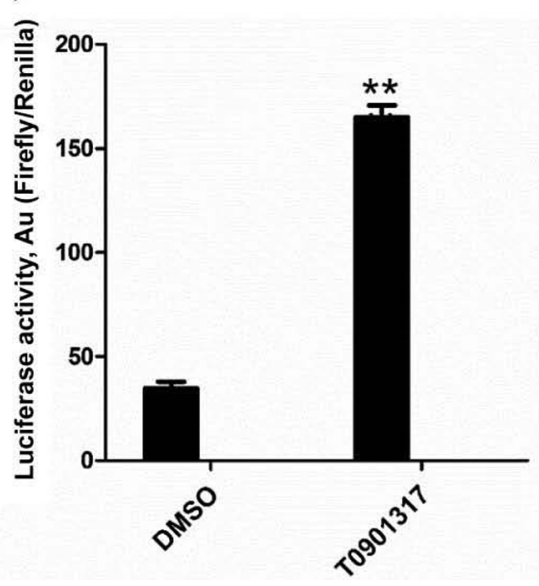

Figure 2. Measurement of sterol regulatory element binding protein-1c (SREBP-1c) luciferase activity. (A) Relative luciferase activity of goat mammary epithelial cells transfected with plasmid pGL3SREBP1c compared with pGL3-basic; (B) effects of SREBP-1 small interfering (si)RNA transfection on SREBP-1c promoter activity; (C) effects of $1 \mu M$ T0901317 (liver X receptor $\alpha$ agonist) or dimethyl sulfoxide (DMSO) on SREBP-1c promoter activity. Values are presented as $\mathrm{LSM} \pm \mathrm{SEM} ;{ }^{* *} P<0.01 ;{ }^{*} P<0.05$. either LXRE suppressed the transcriptional activation of SREBP-1c in response to T0901317. Mutations of both LXRE resulted in an abolishment of the T0901317 activation effect. In addition, T0901317 had no effect on SREBP-1c promoter activity of GMEC transfected with mutations of both LXRE and SRE constructs. These results indicated that LXRE are critical for full activation of SREBP-1c by T0901317. Furthermore, the SRE only enhance this effect and are not indispensable for this regulation.

\section{DISCUSSION}

Since the first purification and characterization of SREBP from nuclear extracts of human HeLa cells (Wang, 1993), their functions in fatty acid and cholesterol synthesis in various cellular systems have been widely investigated (Horton et al., 2003; Chen et al., 2004; Zhao, 2014). Data from a recent study indicated that SREBP-1 regulates the synthesis of glycogen and gluconeogenic genes (Ruiz et al., 2014). Studies in ruminants also provide strong evidence for SREBP-1 as a central player in the pathways regulating mammary fatty acid synthesis (Bionaz and Loor, 2008; Harvatine et al., 2009).

The importance of LXR $\alpha$ in regulating SREBP-1 expression has already been characterized in previous studies (DeBose-Boyd et al., 2000; Repa et al., 2000; Lengi and Corl, 2010). Our results are in accordance with published results and confirmed that full activation of SREBP-1c by T0901317, a specific LXR $\alpha$ agonist, requires the presence of both LXRE and SRE. Although activation of both LXR $\alpha$ and LXR $\beta$ upregulated the expression of SREBP-1c (Repa et al., 2000), the LXR $\beta$ isoform seems mainly involved in ATP-binding cassette, sub-family A (ABC1), member 1 (ABCA1)- and ATP-binding cassette, sub-family G (WHITE), member 1 (ABCG1)-mediated increase of high-density lipoprotein and cholesterol (Quinet et al., 2006), whereas LXR $\alpha$ is the main subtype controlling liver lipogenesis through the activation of SREBP-1c transcription (Peet et al., 1998).

The novel LXRE sequences are composed of 2 inverted DR4-type LXRE at an approximately 30-bp interval, and both binding sites contribute to the activation of SREBP-1c (Yoshikawa et al., 2001). As shown in Figure 1 , the 2 identified LXRE complexes can induce a strong response to LXR activation. By transiently transfecting the $-2,012 \mathrm{bp} /+149 \mathrm{bp}$ fragment into GMEC for luciferase assay, we determined that the promoter activity in the pGL3-SREBP1c-transfected versus pGL3-basic group was significantly higher (Figure 2A). Treatment with T0901317 and SREBP-1 siRNA showed direct regulation of SREBP-1c by LXR and SREBP-1 (Figure 
A)

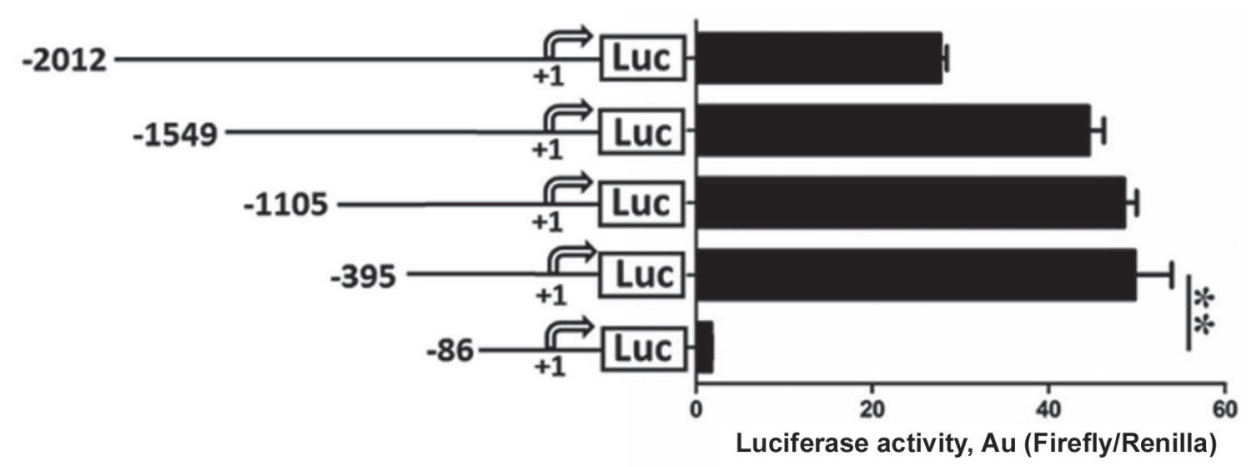

B)

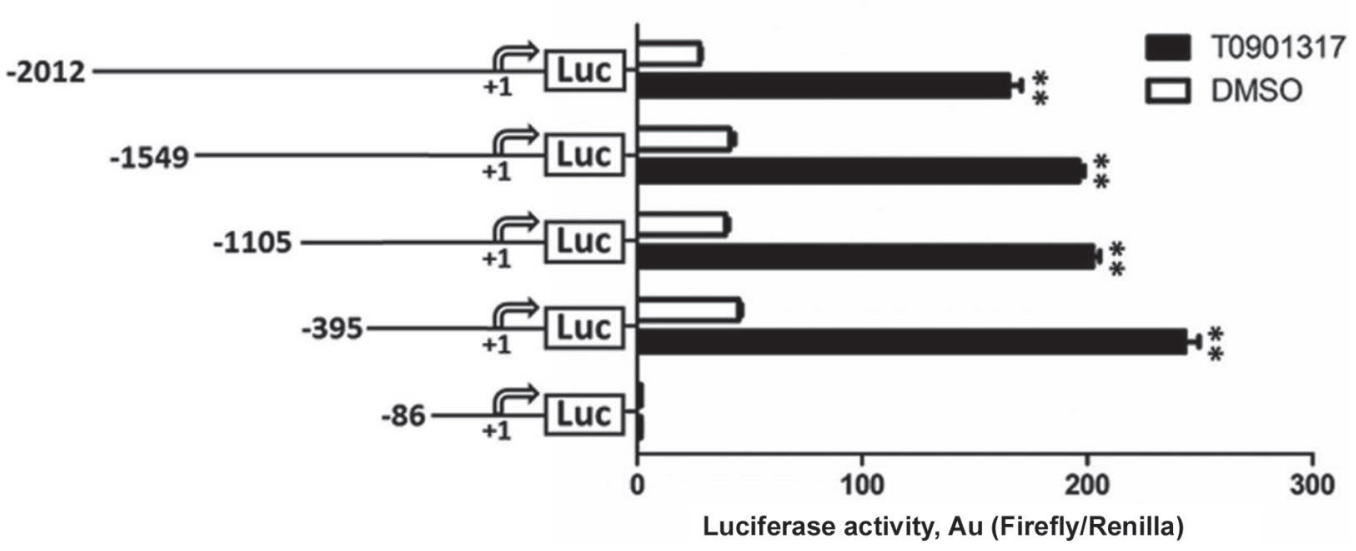

Figure 3. Deletion analysis of goat sterol regulatory element binding protein-1c (SREBP-1c) promoter in goat mammary epithelial cells (GMEC). (A) Relative luciferase activity (firefly:Renilla) after 48 -h transfection with different 5 '-flanking deletion constructs $(-2,012$ bp/ +149 bp, $-1,549 \mathrm{bp} /+149 \mathrm{bp},-1,105 \mathrm{bp} /+149 \mathrm{bp},-395 \mathrm{bp} /+149 \mathrm{bp},-86 \mathrm{bp} /+149 \mathrm{bp}$ ); (B) effects of $1 \mu M$ T0901317 (liver X receptor $\alpha$ agonist) or dimethyl sulfoxide (DMSO) on SREBP-1c promoter activity in GMEC transfected with different 5'-flanking deletion constructs. Values are presented as LSM $\pm \mathrm{SEM} ; * * P 0.01$.

$2 \mathrm{~B}$ and $2 \mathrm{C})$. These results indicate that the cloned fragment was the SREBP-1c promoter.

It was previously reported that insulin activates the human SREBP-1c promoter through SRE motifs (Dif et al., 2006). In fact, a full stimulatory response of SREBP-1c to insulin requires the presence of all sites, with mutations at any sites (SRE or LXRE) in the proximal SREBP-1c promoter reducing the stimulatory effect of insulin by $50 \%$ (Cagen et al., 2005). In our study, the same phenomenon was observed only with the site-directed mutations of the -395 to $+149 \mathrm{bp}$ fragment. The single mutation of LXRE or SRE reduced the activation effect of SREBP-1c by T0901317, and the double mutations of LXRE almost abolished the stimulatory effect of T0901317. However, the reduction effect when each SRE was mutated was not as strong with the $-1,105$ to +149 bp fragment mutations as with -395 to $+149 \mathrm{bp}$. These results further confirmed the hypothesis that the proximal elements are essential for gene expression.
By transiently transfecting the SREBP-1c 5'-flanking truncated luciferase reporter vector into GMEC, we identified a core region that is critically important for SREBP-1c promoter activity (Figure 3A). The changes in stimulatory effects by T0901317 with the different 5 '-flanking truncated fragments further demonstrated that only LXRE in the core region are responsible for the T0901317-induced activation of SREBP-1c (Figure $3 \mathrm{~B})$. The relative promoter activity of SREBP-1c with the simultaneous mutations of both LXRE was not stimulated by T0901317 treatment. In contrast, the mutations in the SRE element still responded to T0901317, with its promoter activity being significantly higher than that of the control group. Taken together, these results demonstrated that full activation of SREBP-1c by LXR $\alpha$, through T0901317, requires the presence of both LXRE located in the -395 to -86 bp region of the SREBP-1c promoter.

The results of the T0901317 treatment after sitedirected mutations further confirmed that LXRE sites 
A)

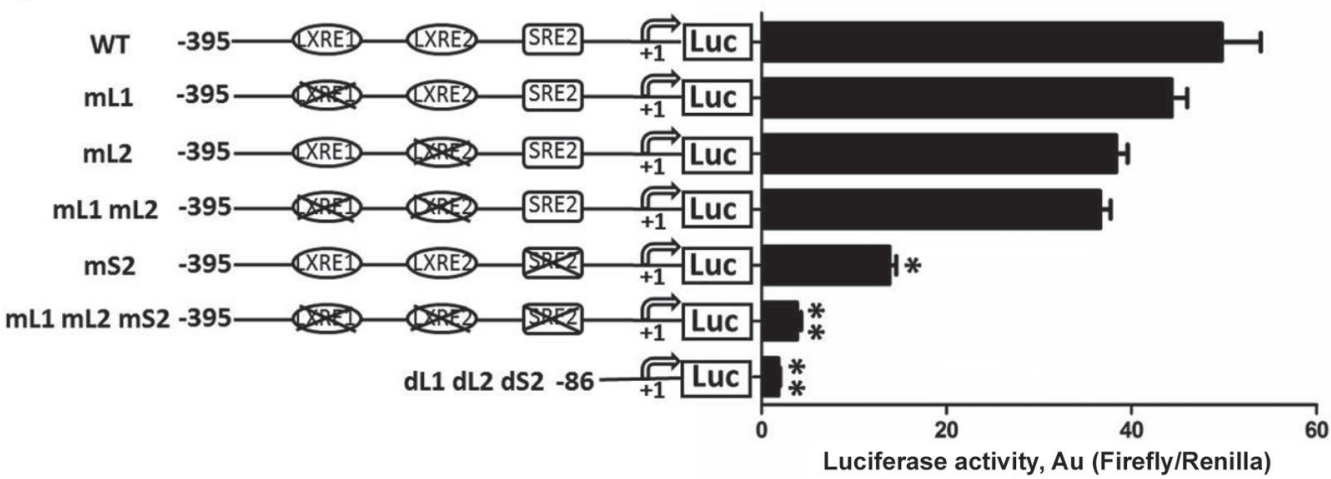

B)

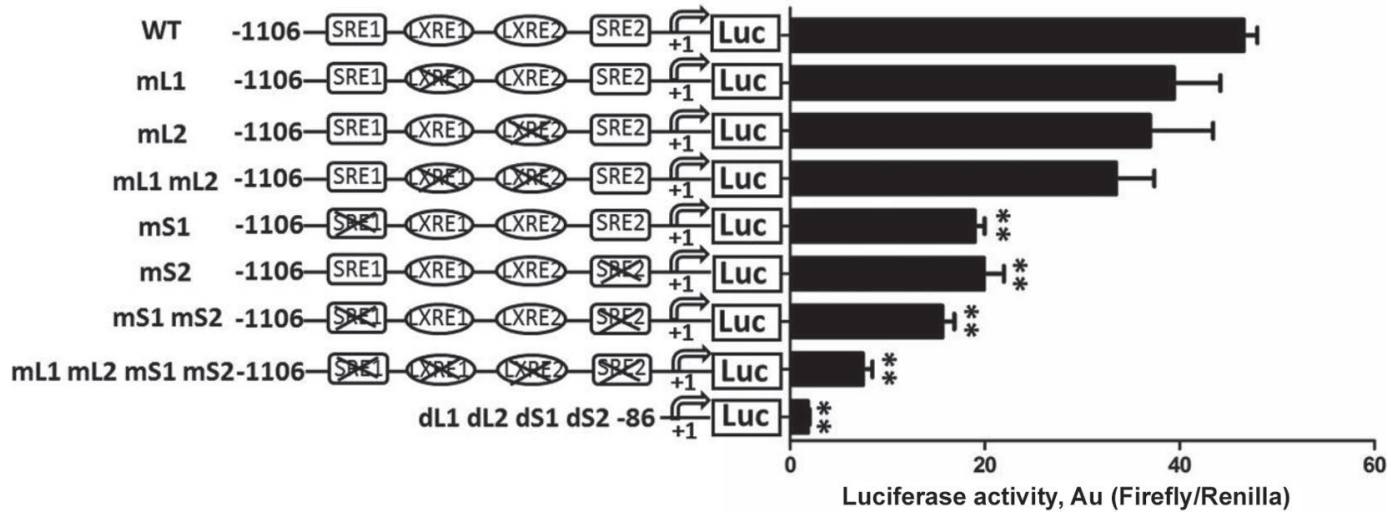

Figure 4. Effects of site-directed mutagenesis on sterol regulatory element binding protein-1c (SREBP-1c) promoter activity. (A) Relative luciferase activity changes after $48 \mathrm{~h}$ of transfection with a $-395 \mathrm{bp} /+149 \mathrm{bp}$ mutated construct of liver X receptor response elements (LXRE) 1 , LXRE2, and sterol response element (SRE)2; (B) relative luciferase activity changes after a 48-h transfection with a $-1,105 \mathrm{bp} /+149 \mathrm{bp}$ mutated construct of LXRE1, LXRE2, SRE1, and SRE2. WT = wild type; $\mathrm{mL} 1=$ LXRE1 mutant; mL2 = LXRE2 mutant; mS1 = SRE1 mutant; $\mathrm{mS} 2=\mathrm{SRE} 2$ mutant. Values are presented as LSM $\pm \mathrm{SEM} ;{ }^{*} P<0.01 ;{ }^{*} P<0.05$

are necessary for the T0901317-mediated activation of SREBP-1c promoter in GMEC (Figure 5A and 5B). A single mutation of either LXRE or double mutations of both LXRE did not significantly reduce SREBP-1c promoter activity, which is in agreement with similar studies (Repa et al., 2000; Yoshikawa et al., 2001; Lengi and Corl, 2010). However, our results are in contrast to those of other studies in which mutations of LXRE sites resulted in a remarkable decrease of human SREBP-1c promoter activity (Dif et al., 2006). We attributed this inconsistency in responses to inherent species differences; for example, to goat and cow being ruminants.

In addition to regulation by LXR $\alpha$, the presence of SRE elements in the goat SREBP-1c promoter implies that mature SREBP-1 protein may bind to SRE to regulate its own expression. Such an "auto-loop" regulatory mechanism has been demonstrated in previous studies (Amemiya-Kudo et al., 2000; Yoshikawa et al., 2001; Takeuchi et al., 2010). Our present study supports this regulatory mechanism. Furthermore, we observed that mutations in either SRE significantly decreased the basal transcription activity of SREBP-1c promoter (Figure 4A and 4B). In fact, promoter activity was even lower with the SRE double mutations (Figure 4B). Those results are in accordance with research into the human SREBP-1c promoter (Dif et al., 2006). In contrast, the present data are inconsistent with published research from bovine mammary cells (Lengi and Corl, 2010). This contradiction may be due to the fragment length used for the site-directed mutagenesis vector construction, because most of the functional binding sites are enriched in the proximal promoter regions (Seo et al., 2009).

However, by transfecting the same luciferase reporter construct of bovine SREBP-1c promoter into both MAC-T cells and bovine mammary epithelial (BMEUV) cells, results from Lengi and Corl (2010) showed that the relative luciferase activity of SREBP-1c was significantly lower in BME-UV cells than in MAC-T cells. Thus, it is possible that the inconsistency in 
response is a result of the use of immortalized cells (MAC-T, BME-UV) compared with primary cells. In fact, previous work demonstrated marked transcriptional differences between MAC-T cells and bovine mammary tissue (Hosseini et al., 2013). Further studies are needed to clarify whether the response we detected for SREBP-1c is unique to goat or also occurs in primary bovine mammary cells.

Previous studies performed with bovine identified 1 SRE site in the proximal region (located in -100 to +1 bp region) of the SREBP-1c promoter (Lengi and Corl, 2010). Although in the human SREBP-1c promoter, 2 SRE sites are located in the -257 to +1 bp region (Dif et al., 2006), the current experiment identified a new SRE site, which is located at $-808 \mathrm{bp}$. Results of site-directed mutagenesis further confirmed that the newly characterized SRE site and the SRE site located in the core promoter region are both important for maintaining SREBP-1c basal transcriptional activity. These results may imply that, for goat SREBP-1c gene regulation, the positive auto-loop circuit regulation is even more important than the LXR-mediated regulation.

We also noted that each mutation of SRE or LXRE site in the proximal region impaired the stimulatory effect of T0901317, indicating the requirement of combinatorial actions of both SRE and LXRE. Cagen et al. (2005) analyzed the rat SREBP-1c promoter and identified NF-Y, Sp1 transcription factor, LXRE, and SRE binding sites, and argued that each of these sites is required for the full stimulatory response of the SREBP-1c promoter to insulin. In the promoter of the human lipin 1 (LPIN1) gene (target gene of SREBP1c), the SRE and NF-Y sites were shown to be essential for the binding of nSREBP-1c protein to its promoter, thus positively activating its expression (Ishimoto et al.,

A)

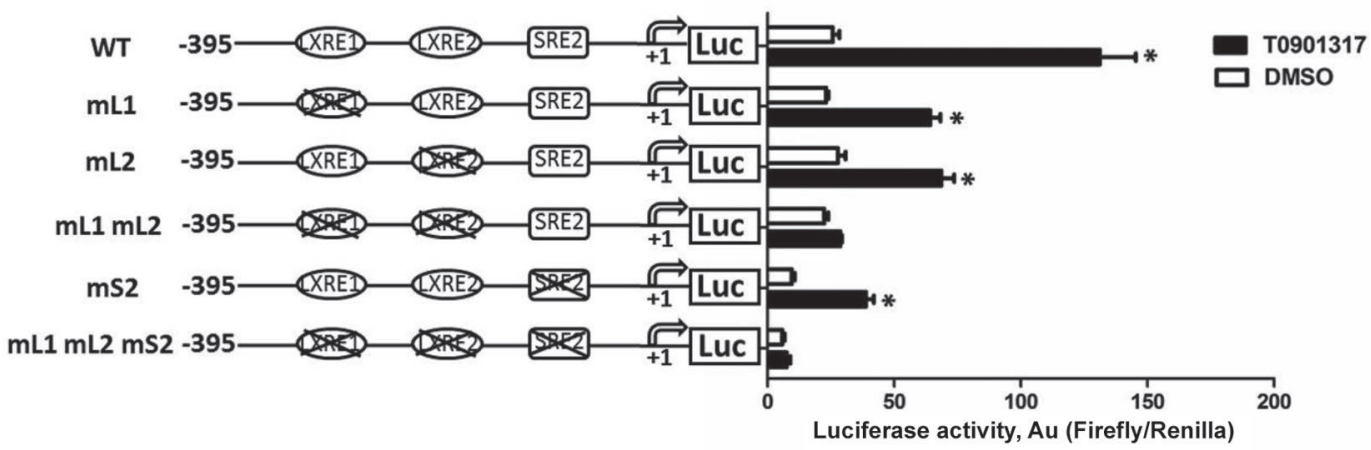

B)

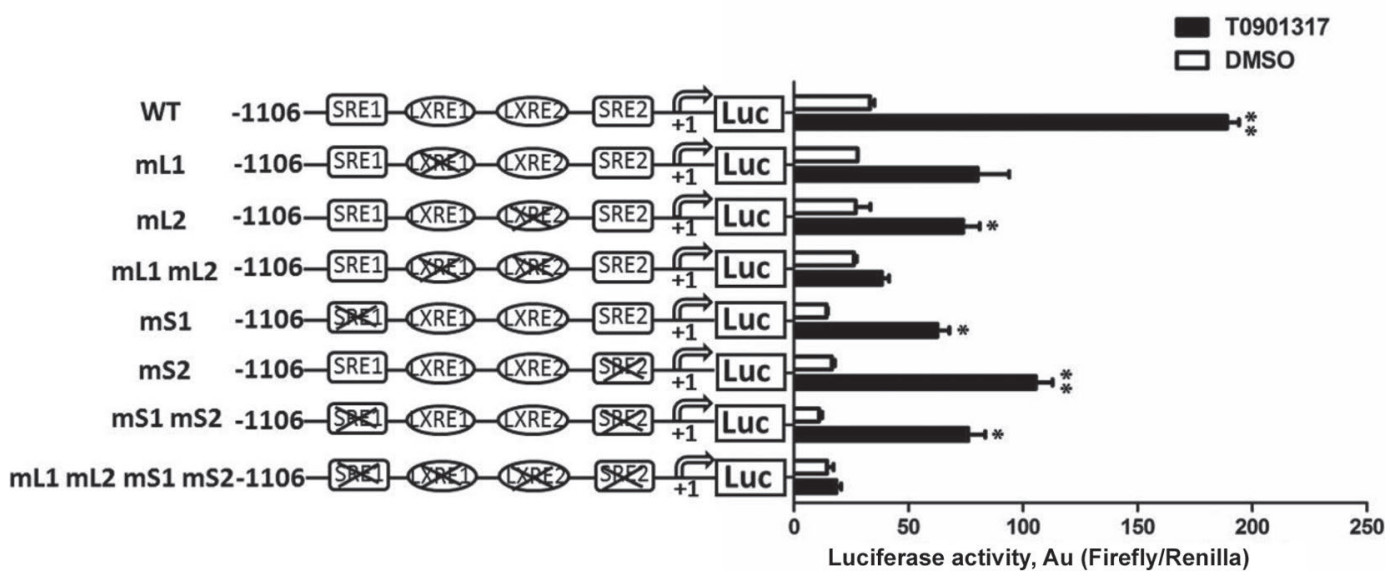

Figure 5. Effects of T0901317 (liver X receptor $\alpha$ agonist) treatment on site-directed mutagenesis promoter activity. (A) Effects of $1 \mu M$ T0901317 or dimethyl sulfoxide (DMSO) treatment on sterol regulatory element binding protein-1c (SREBP-1c) promoter activity in goat mammary epithelial cells (GMEC) transfected with a $-395 \mathrm{bp} /+149 \mathrm{bp}$ fragment plasmid bearing mutations in liver X receptor response elements (LXRE)1, LXRE2, and sterol response element (SRE)2; (B) effects of $1 \mu M$ T0901317 or DMSO treatment on SREBP-1c promoter activity in GMEC transfected with a $-1,105 \mathrm{bp} /+149 \mathrm{bp}$ fragment plasmid bearing mutations in LXRE1, LXRE2, SRE1, and SRE2. WT = wild type; $\mathrm{mL} 1=\mathrm{LXRE} 1$ mutant; $\mathrm{mL} 2=\mathrm{LXRE} 2$ mutant; $\mathrm{mS} 1=\mathrm{SRE} 1$ mutant; $\mathrm{mS} 2=\mathrm{SRE} 2$ mutant. Values are presented as LSM $\pm \mathrm{SEM} ; * * P<$ $0.01 ; * P<0.05$ 
2009). The present study revealed that NF-Y and Sp1 are located in the region near the SRE sites. Although we did not test promoter activity after site-directed mutations of these sites, our results with the T0901317 treatment after mutations of SRE or LXRE, together with the rodent and human data, strongly support the idea that combined actions of SRE, LXRE, Sp1, and NF-Y elements constitute a complex. Such a complex seems essential for the full activation of SREBP-1c and its target genes.

\section{CONCLUSIONS}

We isolated and characterized a 2.0-kbp fragment of SREBP-1c 5'-flanking region, and identified a core promoter region that is essential for the maintenance of its basal transcription activity. Site-directed mutagenesis of LXRE and SRE revealed that the full effects of T0901317 require the presence of both LXRE and SRE. A new SRE element (SRE1) was characterized and a direct role (auto-loop regulation) in maintaining basal transcription activity of SREBP-1c was demonstrated. Overall, our results provide information that could aid in designing studies (in vitro or in vivo) to test the potency of specific nutrients (e.g., long-chain fatty acids) for activating transcriptional mechanisms that could alter goat milk quality.

\section{ACKNOWLEDGMENTS}

This research was jointly supported by the Transgenic New Species Breeding Program of China (Beijing, China; 2014ZX08009-051B) and Special Fund for Agro-scientific Research in the Public Interest (Beijing, China; 201103038).

\section{REFERENCES}

Amemiya-Kudo, M., H. Shimano, T. Yoshikawa, N. Yahagi, A. H. Hasty, H. Okazaki, Y. Tamura, F. Shionoiri, Y. Iizuka, K. Ohashi, J. Osuga, K. Harada, T. Gotoda, R. Sato, S. Kimura, S. Ishibashi, and N. Yamada. 2000. Promoter analysis of the mouse sterol regulatory element-binding protein-1c gene. J. Biol. Chem. 275:3107831085 .

Bionaz, M., and J. J. Loor. 2008. Gene networks driving bovine milk fat synthesis during the lactation cycle. BMC Genomics 9:366.

Brown, M. S., and J. L. Goldstein. 1997. The SREBP pathway: Regulation of cholesterol metabolism by proteolysis of a membranebound transcription factor. Cell 89:331-340.

Cagen, L. M., X. Deng, H. G. Wilcox, E. A. Park, R. Raghow, and M. B. Elam. 2005. Insulin activates the rat sterol-regulatory-elementbinding protein 1c (SREBP-1c) promoter through the combinatorial actions of SREBP, LXR, Sp1 and NF-Y cis-acting elements. Biochem. J. 385:207-216.

Chen, G., G. Liang, J. Ou, J. L. Goldstein, and M. S. Brown. 2004. Central role for liver X receptor in insulin-mediated activation of Srebp-1c transcription and stimulation of fatty acid synthesis in liver. Proc. Natl. Acad. Sci. USA 101:11245-11250.
DeBose-Boyd, R. A., J. Ou, J. L. Goldstein, and M. S. Brown. 2000. Expression of sterol regulatory element-binding protein $1 \mathrm{c}$ (SREBP-1c) mRNA in rat hepatoma cells requires endogenous LXR ligands. Proc. Natl. Acad. Sci U.S.A. 98:1477-1482.

Dif, N., V. Euthine, E. Gonnet, M. Laville, H. Vidal, and E. Lefai. 2006. Insulin activates human sterol-regulatory-element-binding protein-1c (SREBP-1c) promoter through SRE motifs. Biochem. J. 400:179-188.

Eberlé, D., B. Hegarty, P. Bossard, P. Ferré, and F. Foufelle. 2004. SREBP transcription factors: Master regulators of lipid homeostasis. Biochimie 86:839-848.

Espenshade, P. J. 2006. SREBPs: Sterol-regulated transcription factors. J. Cell Sci. 119:973-976.

Harvatine, K. J., Y. R. Boisclair, and D. E. Bauman. 2009. Recent advances in the regulation of milk fat synthesis. Animal 3:40-54.

Horton, J. D., J. L. Goldstein, and M. S. Brown. 2002. SREBPs: Activators of the complete program of cholesterol and fatty acid synthesis in the liver. J. Clin. Invest. 109:1125-1131.

Horton, J. D., N. A. Shah, J. A. Warrington, N. N. Anderson, S. W. Park, M. S. Brown, and J. L. Goldstein. 2003. Combined analysis of oligonucleotide microarray data from transgenic and knockout mice identifies direct SREBP target genes. Proc. Natl. Acad. Sci. USA 100:12027-12032.

Hosseini, A., M. Bionaz, and J. J. Loor. 2013. Transcriptomics comparisons of Mac-T cells versus mammary tissue during late pregnancy and peak lactation. Adv. Dairy Res. 1:103.

Ishimoto, K. H. Nakamura, K. Tachibana, D. Yamasaki, A. Ota, K. Hirano, T. Tanaka, T. Hamakubo, J. Sakai, T. Kodama, and T. Doi. 2009. Sterol-mediated regulation of human lipin 1 gene expression in hepatoblastoma cells. J. Biol. Chem. 284:22195-22205.

Lengi, A. J., and B. A. Corl. 2010. Short communication: Identification of the bovine sterol regulatory element binding protein$1 c$ promoter and its activation by liver $\mathrm{X}$ receptor. J. Dairy Sci. 93:5831-5836

Li, J., J. Luo, H. Xu, M. Wang, J. Zhu, H. Shi, A. B. Haile, H. Wang, and Y. Sun. 2015. Fatty acid synthase promoter: Characterization, and transcriptional regulation by sterol regulatory element binding protein-1 in goat mammary epithelial cells. Gene 561:157-164.

Lin, X.-Z., J. Luo, L.-P. Zhang, W. Wang, H.-B. Shi, and J.-J. Zhu. 2013. miR-27a suppresses triglyceride accumulation and affects gene mRNA expression associated with fat metabolism in dairy goat mammary gland epithelial cells. Gene 521:15-23.

Peet, D. J., S. D. Turley, W. Ma, B. A. Janowski, J. M. Lobaccaro, R. E. Hammer, and D. J. Mangelsdorf. 1998. Cholesterol and bile acid metabolism are impaired in mice lacking the nuclear oxysterol receptor LXR $\alpha$. Cell 93:693-704.

Quinet, E. M., D. A. Savio, A. R. Halpern, L. Chen, G. U. Schuster, J. A. Gustafsson, M. D. Basso, and P. Nambi. 2006. Liver X receptor (LXR)-beta regulation in LXRalpha-deficient mice: Implications for therapeutic targeting. Mol. Pharmacol. 70:1340-1349.

Repa, J. J., G. Liang, J. Ou, Y. Bashmakov, J. M. Lobaccaro, I. Shimomura, B. Shan, M. S. Brown, J. L. Goldstein, and D. J. Mangelsdorf. 2000. Regulation of mouse sterol regulatory element-binding protein-1c gene (SREBP-1c) by oxysterol receptors, LXRalpha and LXRbeta. Genes Dev. 14:2819-2830.

Ruiz, R., V. Jideonwo, M. Ahn, S. Surendran, V. S. Tagliabracci, Y. Hou, A. Gamble, J. Kerner, J. M. Irimia-Dominguez, M. A. Puchowicz, A. DePaoli-Roach, C. Hoppel, P. Roach, and N. Morral. 2014. Sterol regulatory element-binding protein-1 (SREBP-1) is required to regulate glycogen synthesis and gluconeogenic gene expression in mouse liver. J. Biol. Chem. 289:5510-5517.

Seo, Y. K., H. K. Chong, A. M. Infante, S. S. Im, X. Xie, and T. F. Osborne. 2009. Genome-wide analysis of SREBP-1 binding in mouse liver chromatin reveals a preference for promoter proximal binding to a new motif. Proc. Natl. Acad. Sci. USA 106:13765-13769.

Shi, H., J. Luo, J. Zhu, J. Li, Y. Sun, X. Lin, L. Zhang, D. Yao, and H. Shi. 2013. PPAR gamma regulates genes involved in triacylglycerol synthesis and secretion in mammary gland epithelial cells of dairy goats. PPAR Res. 2013:310948.

Shimano, H., J. D. Horton, I. Shimomura, R. E. Hammer, M. S. Brown, and J. L. Goldstein. 1997. Isoform 1c of sterol regulatory 
element binding protein is less active than isoform 1a in livers of transgenic mice and in cultured cells. J. Clin. Invest. 99:846-854.

Shimomura, I., H. Shimano, J. D. Horton, J. L. Goldstein, and M. S. Brown. 1997. Differential expression of exons 1a and 1c in mRNAs for sterol regulatory element binding protein-1 in human and mouse organs and cultured cells. J. Clin. Invest. 99:838-845.

Takeuchi, Y., N. Yahagi, Y. Izumida, M. Nishi, M. Kubota, Y. Teraoka, T. Yamamoto, T. Matsuzaka, Y. Nakagawa, M. Sekiya, Y. Iizuka, K. Ohashi, J. Osuga, T. Gotoda, S. Ishibashi, K. Itaka, K. Kataoka, R. Nagai, N. Yamada, T. Kadowaki, and H. Shimano. 2010. Polyunsaturated fatty acids selectively suppress sterol regulatory element-binding protein-1 through proteolytic processing and autoloop regulatory circuit. J. Biol. Chem. 285:11681-11691.

Wang, X., M. R. Briggs, X. Hua, C. Yokoyama, J. L. Goldstein, and M. S. Brown. 1993. Nuclear protein that binds sterol regulatory el- ement of low density lipoprotein receptor promoter. J. Biol. Chem. 268:14497-14504

Wang, Z., J. Luo, W. Wang, W. S. Zhao, and X. Z. Lin. 2010. Characterization and culture of isolated primary dairy goat mammary gland epithelial cells. Wu Gong Cheng Xue Bao 26:1123-1127.

Yoshikawa, T., H. Shimano, M. Amemiya-Kudo, N. Yahagi, A. H Hasty, T. Matsuzaka, H. Okazaki, Y. Tamura, Y. Iizuka, K. Ohashi, J. Osuga, K. Harada, T. Gotoda, S. Kimura, S. Ishibashi, and N. Yamada. 2001. Identification of liver X receptor-retinoid X receptor as an activator of the sterol regulatory element-binding protein 1c gene promoter. Mol. Cell. Biol. 21:2991-3000.

Zhao, X., Xiaoli, H. Zong, A. Abdulla, E. S. T. Yang, Q. Wang, J. Y. Ji, J. E. Pessin, B. C. Das, and F. Yang. 2014. Inhibition of SREBP transcriptional activity by a boron-containing compound improves lipid homeostasis in diet-induced obesity. Diabetes 63:2464-2473. 